\title{
LOS TESTAMENTOS TRAICIONADOS DE MAYO DEL 68
}

\section{THE BETRAYED TESTAMENTS OF MAY '68}

\author{
Vladimir López Alcañiz \\ International Network for Theory of History
}

\section{RESUMEN}

Este texto se centra en ciertas distorsiones de la memoria de Mayo del 68, sobre todo en aquellas que han producido quienes una vez fueron sus portadores. Además de la atención al objeto de estudio, el artículo pretende ofrecer un sucinto marco teórico para sopesar las razones que pueden conducir a traicionar el legado de un acontecimiento histórico y subrayar la dificultad de mantenerse fiel a él recibiendo creativamente su testamento.

Palabras clave: herencia, memoria, pasado activo, Mayo del 68.

\section{ABSTRACT}

This text focuses on certain distortions of the memory of May '68, especially on those that have been made by those who were once the bearers of that memory. In addition to the attention paid to the object of study, the article aims to offer a concise theoretical framework to ponder the reasons that may lead to betraying the legacy of an historical event and highlighting the difficulty of remaining loyal to the event by creatively receiving its testament.

Keywords: Inheritance, memory, active past, May '68.

\section{SUMARIO}

1.- Introducción. 2.- Cronología de un proceso. 3.- La herencia imposible. 4.- Herederos del acontecimiento. 5.- El porvenir del pasado. 6.- Bibliografía 


\section{Introducción}

¿Qué es un testamento histórico? El legado de un acontecimiento es, primero, lo que podemos aprender de él, aquello que aumenta el espacio de nuestra experiencia: las lecciones que extraemos del pasado, por así decir, aunque estas no puedan trasladarse literalmente al presente; $y$, segundo, es todo lo que podemos activar $y$ hacer nuestro de lo ocurrido, aquello que ensancha el horizonte de nuestra expectativa: todos los combates ganados por la emancipación y la dignidad humanas, pero también, tal vez sobre todo, las esperanzas truncadas, los futuros pasados, las promesas que, precisamente por no haberse cumplido, nos aguardan como un fondo de pasión intacta para que los rescatemos en el instante del peligro. Entre la imaginación y la memoria hay un vínculo inextricable que las semillas del tiempo requieren como agua de mayo.

¿Cómo se traiciona un testamento? Milan Kundera, en el ensayo que ha inspirado el título de este artículo, da algunas pistas al respecto. Existe, por ejemplo, un «espíritu del proceso» -en el sentido kafkiano del término- que consiste en «la reducción del todo a la moral» y que puede conducirnos a olvidar, de una época o un acontecimiento, «todo lo que no es crimen». Semejante selección de lo que merece recordarse jamás hace justicia a lo acaecido. También podemos caer en la tentación, a veces inconsciente, de «evitar los escándalos, de hacer como si no existieran» y arrojar por ende sobre los sucesos extraordinarios «el velo de los lugares comunes» (Kundera, 1994: 241, 245, 157); tratar de sellar la «brecha» con una sutura en falso. Así, la interpretación histórica solo alcanza a depararnos un pasado muerto.

Y hay más. Ahí están las figuras en las que, de acuerdo con Albert $O$. Hirschman, se ampara la «retórica de la reacción», especialmente las de la «perversidad» o la «futilidad» de las transformaciones políticas y sociales abruptas. El primer argumento sostiene que «la tentativa de empujar a la sociedad en determinada dirección resultará, en efecto, en un movimiento, pero en la dirección opuesta»; el segundo, por su parte, afirma que «de una manera o de otra todo pretendido cambio es, fue o será en gran medida de superficie, de fachada, cosmético, y por tanto ilusorio» (Hirschman, 1991: 21, 55). Tales razonamientos no solo pueden esgrimirse ante las transformaciones en curso o por venir, sino también en la reconstrucción retrospectiva de su advenimiento e impacto. Este es el caso del revisionismo histórico, que, como ha sabido ver Jacques Rancière, en el límite significa «la declaración de inexistencia de su objeto», a la que se acerca a través de dos asíntotas: "el no lugar de su causa» y el «casi nada de su efecto» (Rancière, 1993: 49). Volveremos a encontrarnos con estas consideraciones en nuestro camino, que ahora empieza. 


\section{Cronología de un proceso}

Según Pierre Rosanvallon, a partir de los años noventa -quizá incluso una década antes, sobre todo desde una perspectiva global- el panorama ideológico fue transformándose hasta producir un «gran giro» que redefinió «las líneas de fractura intelectuales» en Francia (Rosanvallon, 2018: 299). El movimiento de reorientación del pensamiento empezó en la derecha, como no ocurría desde los años treinta, e impregnó el resto del espectro político. En general, puede decirse que se trató de una vuelta al orden que, teoréticamente, cristalizó en diversas "operaciones retorno»: del humanismo, de la subjetividad, de la razón, de la verdad, etcétera. En particular, cabe añadir que prevaleció el empeñó en negar que realmente pasara algo en los años sesenta y setenta.

En efecto, el proceso a Mayo del 68 constituye uno de los ejes en torno a los cuales pivotó ese "gran giro» del pensamiento. Su singularidad radica en que no es una mera prolongación de la crítica que la derecha conservadora vertió sobre el acontecimiento nada más ocurrir, cuyo mejor ejemplo tal vez sean los artículos de Raymond Aron recogidos en La Révolution introuvable, una obra publicada apenas dos meses después de mayo en la que se calificaba la revuelta sesentayochista de «psicodrama» y «delirio colectivo», entre otras lindezas. De hecho, fue en el seno de cierta izquierda desde donde se empezó a instruir el proceso. El primero en hacerlo fue Régis Debray, antiguo compañero de Fidel Castro y el Che Guevara en Cuba y Bolivia, con su Modeste contribution aux discours et cérémonies officielles du dixième anniversaire, un texto que fija una de las líneas discursivas del antisesentayochismo: la invectiva marxista según la cual Mayo del 68 no socavó la sociedad capitalista, sino todo lo contrario: «La República burguesa celebró su nacimiento en la toma de la Bastilla, un día celebrará su renacimiento en la toma de la palabra del 68» (Debray, 1978: 10). Si Mayo tuvo algún efecto, según esta lectura, este fue más bien perverso.

Semejante crítica, sin embargo, llegaba antes de tiempo y traslucía el resentimiento de un hombre que creía estar haciendo la verdadera revolución en Sierra Maestra hacia los jóvenes protagonistas de Mayo, cuya fama se debía tan solo, a juicio del futuro mitterrandista, a haber escenificado una farsa. Por todo ello, no terminó de cuajar. Mayor éxito cosechó el libro de Luc Ferry y Alain Renaut La Pensée 68 -que resultó ser más bien expresión de la pensée 86. Para conjurar su influencia y su fama, los dos filósofos kantianos emplazaron el pensamiento de los Foucault, Deleuze, Bourdieu, Althusser o Derrida bajo el signo de la repetición, redujeron sus propuestas a la recuperación de algunos señeros filósofos alemanes -especialmente Marx, Nietzsche, Freud y Heidegger-y dirigieron contra ellos una acusación genérica: «El pensamiento francés de los años 68 escogió resueltamente el partido del antihu- 
manismo» (Ferry y Renaut, 1985: 18), lo que significa que hicieron una apuesta por la muerte del sujeto y de la verdad que a la postre resultó perdedora, según los autores. Sin novedad en el frente, la mayor debilidad de la pensée 68 sería pues su futilidad.

Pero es el libro de Ferry y Renaut el que no puede escapar a su mayor debilidad: la elección de los protagonistas, en su mayoría ajenos al 68 o incluso críticos con el acontecimiento, acaba por no decir apenas nada del pensamiento que realmente dio forma a Mayo del 68. Tendría que llegar el trigésimo aniversario de los hechos, en 1998, para que el proceso encontrara su pieza clave: la obra del sociólogo Jean-Pierre Le Goff Mai 68, I'héritage impossible. Pronto nos detendremos en ella, pero quedémonos ahora unos años antes. Concretamente, cuando Pierre Nora concluyó el monumental proyecto editorial sobre Les Lieux de mémoire (1984-1992) con una reflexión sobre la «era de la conmemoración» en la que dedicaba a Mayo del 68 unas duras palabras que merecen ser citadas in extenso:

Mayo de 1968 [...] encarna, sin quererlo, el imperio de la memoria conmemorativa. [...] Todos se preguntaron después qué fue lo que pasó realmente. No hubo revolución, incluso nada tangible y palpable, sino, muy a pesar de los actores, el resurgimiento incoercible y el festival esplendoroso del legendario completo de todas las revoluciones: las del siglo xx y hasta del siglo XIX francés, con la juventud de las escuelas que recordaba 1848, las barricadas de la Comuna, las marchas del Frente popular, el recuerdo vivo aún de la Resistencia; la de los sóviets de Petrogrado y de la toma de poder leninista; las del tercer mundo, de China a Cuba. No se terminaría nunca de contabilizar la fantasmagoría histórica de la que Mayo del 68 fue el recapitulativo puramente simbólico. Los soixante-huitards [...] querían actuar, [pero] lo único que hicieron fue celebrar, en un último festival y una reviviscencia mimética, el fin de la Revolución. El acontecimiento no tiene otro sentido más que el conmemorativo. Mayo del 68 incluso se adelantó al Bicentenario, al conmemorar a su pesar el fin de lo que supuestamente 1989 debía celebrar (Nora, 1992: 979-980).

Es cierto que Pierre Nora nunca fue un sesentayochista, pero este pasaje retrata a la perfección dos flancos del ataque al 68: primero, la afirmación de que no fue lo que pretendía ser, es decir, una revolución; y segundo, la negación de su potencial para el futuro, esto es, para nosotros. Más sorprendente es que, en alguna medida, comparta esta última consideración uno de los líderes del movimiento, Daniel Cohn-Bendit. En un libro de entrevistas publicado en 2009 y titulado explícitamente Forget 68, sostiene: «jEl 68 ha terminado! Eso no quiere decir que ese pasado esté muerto, sino que está sepultado bajo cuarenta toneladas de adoquines que, desde entonces, han labrado y cambiado el mundo. El mundo de hoy ya no es en absoluto el del 68» (Cohn-Bendit, 2009: 118) y seguir pensando en función de las categorías de entonces no hace avanzar a nadie. 
Esta es la visión que ha hecho suya, como cabía esperar, el actual presidente de la República francesa Emmanuel Macron, quien reconoce que el 68 tenía sus fundamentos y su actualidad entonces, pero considera que fue solo un instante histórico y, como tal, pertenece al pasado. Tras la desactivación del pasado se esconde el peligro de la tergiversación y la banalización. El salto lo ha dado recientemente un columnista del Financial Times al afirmar que los actuales manifestantes contra las políticas del gobierno francés «se aferran al pasado, no al futuro» y que, en este sentido, es Macron, que sí mira al porvenir, «el legítimo heredero del espíritu de 1968» (Stephens, 2018).

No deberíamos tomarnos a la ligera estas lecturas superficiales de la historia, por más que de buenas a primeras nos parezcan irrisorias. El abuso de la memoria y la deformación del pasado son una de las principales armas del autoritarismo, como ha mostrado de forma elocuente Bruno Tertrais en La venganza de la historia. Bajo la presidencia de François Hollande, la «Manif pour tous» en contra del matrimonio homosexual quiso verse como el reverso del 68: sirviéndose de su activismo, recuperó la retórica reaccionaria sobre el declive de la moral, la autoridad y la familia, exactamente lo mismo que está haciendo el movimiento antiabortista en Estados Unidos. Por otro lado, la cruzada contra la «ideología de género» que impugna la emancipación de las mujeres ha encontrado en el contexto internacional, donde Putin y Trump representan la exaltación de una virilidad agresiva, el apoyo para atacar las conquistas sociales del feminismo desde los años sesenta. Ante semejante movilización del pasado para controlar el futuro, recobrar la memoria de Mayo es un paso indispensable para liberarlo.

\section{La herencia imposible}

Antes de presentar la defensa de Mayo, veamos la mejor acusación. Jean-Pierre Le Goff no tiene ninguna duda de que el 68 es un acontecimiento decisivo, uno que guarda una relación singular con la historia y la memoria oficiales: elude la conmemoración y se resiste a reducirse a una panoplia de héroes. Aunque no por ello esquiva la cuestión cardinal que debe dirigirse a todo acontecimiento: ¿̇cuál es su legado? $O$, más concretamente: ¿̇qué nos ha dejado para pensar de otro modo el mundo en común e imaginar un futuro en el que tenga cabida la esperanza de emancipación? Cuando se refiere al 68, la pregunta es tanto más acuciante cuanto que, después de Mayo, se han deshilachado los relatos de emancipación y se ha ocluido la imaginación del futuro, en ocasiones -y ahí está la paradoja- en nombre de los ideales del propio acontecimiento. El 68 genera su propia sombra, que lo devora.

¿Por qué ha ocurrido esto? Según Le Goff, el motivo principal hay que buscarlo en el hecho de que los años contestatarios no solo desembocaron en el fin del mito revolucionario, 
sino que socavaron los fundamentos éticos de lo político hasta el punto de poner en duda la posibilidad de su reconstrucción. En este sentido, constituyen una «herencia imposible» que nos deja con una inquietante pregunta (Le Goff, 1998: 20): ¿əabe dentro del horizonte de Mayo la búsqueda actual de una renovación política y cultural? De la respuesta depende que el 68 sea todavía un pasado presente o solo un futuro pasado.

Y la que ofrece el sociólogo no es muy benevolente, pero tiene la virtud de darnos elementos sobre los que reflexionar y constituye una buena referencia con la que confrontarnos y refrenar cualquier tentación de mitificar Mayo del 68. ¿Qué nos dice Le Goff? Fundamentalmente, que las reivindicaciones de Mayo conducen a un impasse infranqueable. Y ello en dos sentidos. Primero: para los principales actores del 68, Mayo es como «un tiempo suspendido en su máximo nivel de intensidad y abierto a todas las posibilidades». No obstante, la exigencia de libertad y autonomía absolutas, sin referentes, dependencias ni anclajes, tiene su corolario extremo en «la negación de todo sentimiento de deuda hacia las generaciones pasadas y futuras», lo cual supone una quiebra de la transmisión que pone en un brete «la idea de vínculo de filiación, de memoria y porvenir comunes» (Le Goff, 1998: $471,461)$. La libertad absoluta incluye irremediablemente la libertad respecto del pasado, un gesto típicamente revolucionario que lleva fácilmente -y las más de las veces, fatalmentea la desmesura, a la hýbris, ese funesto motor de la historia para Heródoto.

Y segundo: los sesentayochistas no solo pretenden romper con el pasado, también apropiarse de él. Por eso activan el pasado, tanto el político como el poético: la inspiración proteica de Rimbaud y Breton convive con el cuestionamiento de la monarquía -ahora encarnada en Charles de Gaulle- de 1789, las barricadas de 1848, la festividad de 1871, el lenguaje de 1917 y las ocupaciones de 1936. A pesar de ello, para Le Goff el imaginario revolucionario no encuentra el modo de arraigarse en el presente. Antes bien, la condensación de la herencia revolucionaria conduce a su implosión. En efecto, al quererse herederos de las luchas más importantes de la historia, ponen muchas dificultades a las posibilidades de filiación, ya que todo palidece ante ellas. La tradición revolucionaria, en su repetición como farsa, conduce a un callejón sin salida que contribuye a descomponer el izquierdismo político. Al querer salir de él, «el heredero imposible de Mayo del 68 se vuelve imprecatorio», pero su denuncia constante de todo lo instituido solo enmascara la indigencia de su reflexión y pervierte el espíritu crítico. El «no» de la denuncia y la queja ya no está sostenido por un «sí» originario que constituya el vínculo de confianza que puede unirnos al mundo (Le Goff, 1998: 474).

¿Qué decir ante ello? En primer lugar, hay que reconocer que Le Goff concede que existen dimensiones de Mayo que permanecen como una potencialidad incumplida del pasa- 
do y, por tanto, reclaman un trabajo de reconstrucción y reprise. En segundo lugar, si hoy todavía queremos hacer balance y asumir el testamento de Mayo, debemos tener en cuenta sus extremos, pero también tenemos que saber replicar que estos no son representativos de todo el movimiento. Y, particularmente sobre la autonomía y la libertad, tenemos que recordar que, entonces, tales reivindicaciones siempre estuvieron acompañadas de la búsqueda de nuevas formas de comunidad e igualdad. Por último, no podemos pasar por alto que la recuperación de la tradición revolucionaria no implica necesariamente su repetición. Un ejemplo: según la interpretación de Jacques Baynac, las barricadas que se erigen en el 68 no tienen ninguna utilidad defensiva ni son una escenificación nostálgica y anacrónica de los fantasmas de las revoluciones pasadas. Se inscriben en una tradición política de invención de nuevas formas de utilizar el espacio y el tiempo. Son, en el fondo, barricadas de tiempo suspendido, liberado de la relojería del capital. «Hacen perder el tiempo al Poder y ganan tiempo para el no poder» (Baynac, 1978: 393), para la horizontalidad radicalmente democrática de la que el 68 recoge la antorcha. Recuperaremos este hilo al salir del laberinto.

\section{Herederos del acontecimiento}

El recuerdo del 68 ha sido rescrito y sobrescrito por multitud de relatos, discursos, memorias y análisis, que han seguido estrategias de lo más variadas para alcanzar sus fines, no siempre justos con su objeto de interés. La sedimentación de tantos estratos interpretativos hace que hoy sea necesario un esfuerzo para penetrar el sentido de esa revuelta, pues incluso algunos de quienes la vivieron parecen haberlo olvidado. De todos ellos, solo algunos han tratado de permanecer fieles al acontecimiento a través de nuevas formas de compromiso y pasión democrática. Otros, sin embargo, han renunciado a la pasión e incluso han renegado de ella, quizá sin darse cuenta de que, con ese gesto, estaban renunciando también a la memoria, pues hay dimensiones del recuerdo a las que solo puede accederse con la pasión intacta. La primera pregunta que surge al respecto es esta: ¿por qué tantos sesentayochistas han rechazado, incluso de plano, la radicalidad política y el compromiso? Pero, a continuación, debemos formular esta otra: ¿qué podemos aprender de su actitud? Desde luego, son cuestiones que nos afectan, quizá porque intuimos que nuestro lento presente no dará paso a un futuro distinto si no elaboramos la memoria del 68 y asumimos su legado.

¿Cómo hacerlo? Este breve excurso nos ayudará a elucidarlo. Un acontecimiento histórico -que, como es sabido, se distingue de un suceso cualquiera- desgarra la trama de la realidad del tal forma que impele a quienes la viven a decidir una nueva manera de ser. Así, por ejemplo: la política y la historia tras la revolución francesa, la música contemporánea tras 
el dodecafonismo de Schoenberg, la ciencia moderna tras la irrupción de la física galileana - la propia intimidad tras una historia de amor son acontecimientos que exigen, si se quiere serles fiel, que desde su advenimiento cada actualidad se refiera a ellos y se piense en función de ellos. Lo cual, dado que el acontecimiento posee un carácter generativo que rompe con las leyes antes al uso, «obliga a inventar una nueva manera de ser y de actuar» después de él (Badiou, 2004: 71). Y no es fácil hacerlo: aquellos a los que un acontecimiento niega, margina o envejece tienden a su vez a negar o marginar el acontecimiento en sus relatos, 0 a arrojar sobre él «el velo de los lugares comunes».

En definitiva, el acontecimiento contiene la exigencia de hacerse cargo de él, «de la memoria, la herencia y las generaciones», como escribió Derrida. Y esa carga, al caminar con nosotros, genera un principio de responsabilidad hacia el futuro. Siguiendo al filósofo francés, tenemos, por un lado, la necesidad de una «política de la memoria»; y por el otro, la sabiduría de que ninguna política ni ninguna justicia «parece posible o pensable sin un principio de responsabilidad» que vaya «más allá de todo presente vivo» (Derrida, 1995: 12-13).

Hacia atrás, la herencia. Gracias a pensadores como Hans-Georg Gadamer o Jacques Derrida sabemos que recibir una herencia no es un acto pasivo: recibir algo que se nos da y retenerlo sin más. Antes al contrario, para que haya herencia ha de haber decisión, uno ha de hacerse cargo de ella eligiendo: seleccionándola, interpretándola, incluso traicionándola. La lealtad a la herencia conlleva necesariamente un cierto grado de infidelidad que nos aleje de la mera aquiescencia, de la repetición compulsiva o de la fosilización de lo heredado. Para poder heredar, y para que la herencia no sea un fardo, hay que recibirla creativamente, porque ella ha de abrirnos una ventana al futuro, darnos posibilidades, ser una promesa y no un programa, algo que nos comprometa sin obligarnos. $O$, parafraseando al poeta René Char, una herencia sin testamento.

Hacia delante, el porvenir. Cuando la herencia nos alcanza, pone en cuestión el futuro, lo que está por venir. De modo que, aunque provenga del pasado, la herencia está siempre vuelta hacia el futuro. Va hacia él, y por eso, también viene de él. Derrida pone de relieve la inadecuación a sí mismo del presente y nos invita a una experiencia del pasado como porvenir. Si partimos desde un lugar, ese sitio asigna la posición con la que estamos comprometidos, y si hay "compromiso o asignación, inyunción o promesa, el "desde" marca un lugar y un tiempo que nos preceden, sin duda, pero para estar tanto delante de nosotros como antes de nosotros» (Derrida, 1995: 31 ).

Visto así, el 68 no está solo detrás de nosotros, como herencia sin testamento, sino también ante nosotros, como exigencia y desafío: porque no solo nos lega recuerdos, sino sobre todo deseos. 


\section{El porvenir del pasado}

El hervidero de los sesenta se proyecta en direcciones que aún hoy condicionan las orientaciones del pensamiento. Plantea cuestiones irresueltas cuyo fondo todavía da forma a nuestras reflexiones. Como una radiación de fondo cósmica, sus ondas nos recuerdan el acontecimiento de una explosión. Rastrearlas es por tanto una tarea que nos incumbe. No por curiosidad, o no principalmente, sino por un compromiso enteramente histórico, que asuma que en la investigación está en juego la agudeza de nuestra propia mirada.

En los años sesenta se localiza el borde de nuestro tiempo. Tras su conjura en falso, los problemas entonces abiertos, que fueron subrepticiamente diferidos y han permanecido en suspenso, hoy regresan y nos requieren. Nos asedian. Estamos todavía en la órbita del acontecimiento, por eso la sola cronología no puede medir la distancia que nos separa de él. Esa distancia es una extraña mezcla de lejanía y cercanía que no podemos ni debemos abolir. Tenemos que trabajar con ella, siendo conscientes de que nuestro trabajo no es exterior al acontecimiento, porque se inscribe en su estela, pero también de los estratos de interpretación que se interponen entre él y nosotros.

En las décadas que siguieron al acontecimiento, su recepción estuvo marcada por el signo de la negación. Hoy en día, sin embargo, carece de sentido seguir insistiendo en los testamentos traicionados. Ni el rechazo, ni la mitificación, ni la melancolía pueden ofrecernos claves de lectura sugerentes, prometedoras. Lo que nos exige nuestro tiempo no es, por consiguiente, un mero retorno -un gesto a menudo vinculado al deseo de una vuelta al orden- sino una reprise, una reformulación abierta y crítica de las cuestiones suscitadas entonces cuya respuesta permanece aún en suspenso. Pues esa suspensión es la que determina la forma de nuestro presente en vilo y nos indica, así, la tarea de nuestro tiempo.

Para terminar, señalaré tres aspectos de la herencia silenciosa de Mayo con los que todavía podemos $-y$, seguramente, debemos- dialogar. El primero es el lazo entre la cultura, el arte y la vida cotidiana. Si hay algo que permanece latente de la transgresión fronteriza del movimiento sesentayochista y que reclama nuestra atención es la articulación inédita entre conocimiento y existencia, teoría y práctica, arte y vida, saber y poder, probablemente fruto de la coincidencia entre la historia y la acción. Con el fin de subvertir efectivamente el orden social y, como quería Rimbaud, «cambiar la vida», la teoría no puede permanecer encerrada dentro de los muros de la academia, sino que debe acompañar a la práctica. Más aún, la teoría tiene que comprenderse a sí misma como una forma de práctica. Por su parte, tampoco el arte puede permitirse el lujo de convertirse en un objeto de consumo o fosilizarse en un museo. Al contrario, debe participar en lo social y, más que representar la realidad, presentar la 
apertura de posibilidades que se produce en ella cuando lo poético anima lo práctico. Hoy, cuando parece que lo sabemos todo y no podemos nada, como ha diagnosticado Marina Garcés, haríamos bien en tratar de rescatar el potencial de las conexiones que Mayo ensayó.

El segundo es el vínculo entre estudiantes y obreros. En el agrietamiento de las fronteras entre trabajadores manuales e intelectuales, nacionales y extranjeros, se perfila una política que no acepta dejar a cada uno en su lugar, que persigue trazar trayectorias originales y fraguar reuniones imposibles entre gentes con poco en común. Porque las personas no se definen por sus identidades anteriores, sino por sus actos durante la revuelta. Unos actos que trastornan las demarcaciones sociales, espaciales y temporales, que impugnan el reparto de lo sensible, y crean nuevos lazos y espacios de coordinación. La política emancipatoria no parte de la herencia de la identidad, sino de la conquista de la igualdad. En un momento, como el nuestro, en el que la crisis parece exorcizarse a través de la xenofobia, conviene tenerlo muy presente.

El tercero es la toma de la palabra. «El último mayo, se ha tomado la palabra como se tomó la Bastilla en 1789», escribió a los pocos meses $-y$, contrariamente a Debray, sin cinismo- Michel de Certeau (1968: 40). En efecto, en el 68 voces hasta entonces silenciadas ocuparon la escena. $Y$, aunque en ese preciso momento no todas pudieron escucharse, sí permaneció, como legado, la necesidad del reconocimiento público y el debate colectivo como medios para proseguir la búsqueda de nuevos derechos. Esto fue especialmente importante para los movimientos sociales de los años setenta, por ejemplo el combate de las mujeres por la igualdad real -todavía inacabado-, que se benefició de las renovadas demandas de participación y de la sensibilidad que otorgó un sentido político a ámbitos que hasta entonces no parecían tenerlo, como la vida privada.

Todo historiador comprometido debería atender la llamada de este pasado. Si decide hacerlo, me parece que, al menos, su tarea ha de cumplir con estas condiciones. En primer lugar, debe rescatar el 68 no solo del olvido, sino sobre todo de esa «memoria-pantalla», en palabras de Boris Gobille, sedimentada desde hace años, que ofrece de él una versión reducida, particularmente a causa de sus supuestas consecuencias: individualismo, presentismo, incluso neoliberalismo. Como lo expresaría el gran historiador E. P. Thompson, hay que rescatar el acontecimiento de la «inmensa condescendencia» de la posteridad. A continuación, debe restituir al 68 la temporalidad que le es propia, es decir, la del acontecimiento, y por tanto conjugarlo todavía en presente, puesto que todo acontecimiento prolonga su existencia pretérita a través de una duradera insistencia. Y, por último, no debe ocultar sus virtualidades radicalmente democráticas. Todo ello con el fin de asumir y traspasar a las siguientes generaciones ese testamento que no pertenece a nadie, y por tanto nos concierne a todos. 


\section{Bibliografía}

BADIOU, Alain (2004). La Ética. Ensayo sobre la conciencia del mal, Ciudad de México: Herder. BAYNAC, Jacques (1978). Mayo del 68: la revolución de la revolución, Madrid: Acuarela \& Antonio Machado, 2016.

Certeau, Michel de (1968). La Prise de parole et autres écrits politiques, París: Seuil, 1994.

Conn-Bendit, Daniel (2009). Forget 68. Entretiens avec Stéphane Paoli et Jean Viard. París: Éditions de l'aube.

DeBray, Régis (1978). Modeste contribution aux discours et cérémonies officielles du dixième anniversaire, París: Maspero.

DerRIDA, Jacques (1995). Espectros de Marx. El estado de la deuda, el trabajo del duelo y la nueva Internacional, Madrid: Trotta.

Ferry, Luc y Renaut, Alain (1985). La Pensée 68. Essai sur l'anti-humanisme contemporain, París: Gallimard.

HiRsChman, Albert O. (1991). Retóricas de la intransigencia, Ciudad de México: FCE.

KUnDERA, Milan (1994). Los testamentos traicionados, Barcelona: Tusquets.

LE Goff, Jean-Pierre (1998): Mai 68, I'héritage impossible, París: La Découverte, 2006.

NORA, Pierre (1992). «L'ère de la commémoration» en Nora, Pierre (ed.) (1992). Les Lieux de mémoire, t. III, Les France, vol. 3, De l'archive à l'emblème, París: Gallimard, pp. 977-1012.

RANCIÈRE, Jacques (1993). Los nombres de la historia. Una poética del saber, Buenos Aires: Nueva Visión.

Rosanvalon, Pierre (2018). Notre histoire intellectuelle et politique 1968-2018, París: Seuil. StePHeNS, Philip (2018). «Emmanuel Macron is the rightful heir to the spirit of 1968» en Financial Times, 19 de abril. Disponible en: https://www.ft.com/content/df8e7c604305-1 1e8-803a-295c97e6fd0b (Fecha de consulta: 15 de septiembre de 2018).

Recibido el 15 de octubre de 2018

Aceptado el 23 de diciembre de 2018 BIBLID [1 139-1219 (2018) 24: 17-27] 